\title{
Gravitational Instability of Rotating Viscoelastic Partially Ionized Plasma in the Presence of an Oblique Magnetic Field and Hall Current
}

\author{
M. F. El-Sayed ${ }^{1}$ and R. A. Mohamed ${ }^{2}$ \\ ${ }^{1}$ Department of Mathematics, Faculty of Education, Ain Shams University, Heliopolis, Roxy, Cairo 11757, Egypt \\ ${ }^{2}$ Department of Physics, Faculty of Education, Ain Shams University, Heliopolis, Roxy, Cairo 11757, Egypt
}

Correspondence should be addressed to M. F. El-Sayed, mfahmye@yahoo.com

Received 29 January 2011; Accepted 17 March 2011

Academic Editor: P. M. Mariano

Copyright (C) 2011 M. F. El-Sayed and R. A. Mohamed. This is an open access article distributed under the Creative Commons Attribution License, which permits unrestricted use, distribution, and reproduction in any medium, provided the original work is properly cited.

\begin{abstract}
The gravitational instability of a rotating Walters $\mathrm{B}^{\prime}$ viscoelastic partially ionized plasma permeated by an oblique magnetic field has been investigated in the presence of the effects of Hall currents, electrical resistivity, and ion viscosity. The dispersion relation and numerical calculations have been performed to obtain the dependence of the growth rate of the gravitational unstable mode on the various physical effects. It is found that viscosity and collision frequency of plasma have stabilizing effects, while viscoelasticity and angular frequency of rotation have destabilizing effect; the electrical resistivity has a destabilizing effect only for small wavenumbers; the density of neutral particles and the magnetic field component in $z$-direction have stabilizing effects for wavenumbers ranges $k<5$ and $k<10$, respectively; the Hall current has a slightly destabilizing effect. Finally, the inclination angle to $z$-direction has a destabilizing effect to all physical parameters.
\end{abstract}

\section{Introduction}

The gravitational instability problem of an infinite homogenous medium was first considered by Jeans [1]. According to Jeans' criterion, an infinite homogenous self-gravitating atmosphere is unstable for all wavenumbers $k$ less than Jeans' wavenumber $k_{j}=\sqrt{G \rho} / S$, where $\rho$ is the density, $S$ is the velocity of sound in the gas, and $G$ is the gravitational constant. This problem has been studied by several authors under varying assumptions of hydrodynamics and hydromagnetics, and a comprehensive account of these investigations has been given by Chandrasekhar [2] in his monograph on problems of hydrodynamic and hydromagnetic stabilities. He showed that Jeans' criterion remains unaffected by the separate or simultaneous presence of uniform rotation and uniform magnetic field. The combined effects of uniform rotation, Hall currents, finite conductivity, and finite Larmor radius on gravitational instability have been studied by Bhatia [3]. Yu and Sanborn [4] studied the internal gravitational instability in a stratified anisotropic plasma. Bhatia [5] and also Barbian and Rasmussen [6] studied the gravitational instability of a rotating anisotropic plasmas. Ariel [7] studied the gravitational instability of a rotating anisotropic plasma with Hall current effect.

In cosmic physics, there are several situations such as chromosphere, solar photosphere, and in cool interstellar cloud where the plasma are frequently not fully ionized but may instead be partially ionized so that the interaction between the ionized fluid and the neutral gas becomes important. The importance of such collisions between ionized fluid and neutral gas on the ionization rate in these regions have been pointed by Mamun and Shukla [8]. They studied a new magnetic Jeans instability in a non-uniform partially ionized plasma. Pandey et al. [9] studied Jeans instability of an inhomogeneous streaming dusty plasma. 
Daughten et al. [10] studied interchange instabilities in a partially ionized plasma. Mamun [11] investigated the effect of temperature and fast ions on gravitational instability in a self-gravitating magnetized dusty plasma. Mamun and Shukla [12] studied instabilities of self-gravitating dusty clouds in magnetized plasmas. The magnetoplasma stability problems have been recently studied by several authors, for example Mamun and Shukla [13], De Juli et al. [14], Cramer and Verheest [15], Azeem and Mirza [16], and El-Sayed and Mohamed [17]. There are many viscoelastic fluids that cannot be characterized by Maxwell's or Oldroyd's constitutive equations. One of such classes of viscoelastic fluids is Walters $\mathrm{B}^{\prime}$ liquid. As a result, the usual viscous term in the equation of motion in the case of viscoelastic Walters $\mathrm{B}^{\prime}$ fluid is replaced by the resistive term ( $v-$ $\left.v^{\prime} \partial / \partial t\right) \mathbf{u}$ where $v$ and $v^{\prime}$ are the viscosity and viscoelasticity of the Walters $\mathrm{B}^{\prime}$ fluid. This type of viscoelastic fluids have been investigated by Sharma and Sunil [18] in the stability of two superposed Walters B' viscoelastic liquids. Kumar [19] studied stability of two superposed Walters $\mathrm{B}^{\prime}$ viscoelastic fluid particle mixtures in porous medium. He [20] also studied the thermal convection in Walters B' viscoelastic fluid permeated by suspended particles in porous medium.

In all the investigations, the prevalent magnetic field is assumed to act either along the horizontal or the vertical direction. Chhajlani and Vyas [21] have studied the KelvinHelmholtz instability problem in an oblique magnetic field. Khan and Bhatia [22] considered the effects of finite resistivity and collisions with neutrals on Rayleigh-Taylor instability of a stratified plasma. Ali and Bhatia [23] have studied the gravitational instability of a partially ionized plasma in an oblique magnetic field with the effects of Hall currents, magnetic resistivity, and ion viscosity. Therefore, it would be of importance here to examine the effects of viscosity, viscoelasticity, Hall current, rotation, and finite conductivity for two components Walters $\mathrm{B}^{\prime}$ viscoelastic partially ionized plasma in a uniform oblique magnetic field. This problem, to the best of our knowledge, has not been investigated yet.

\section{Problem Formulation and Perturbation Equations}

Let us consider the motion of an infinite homogeneous finitely conducting viscoelastic Walters $\mathrm{B}^{\prime}$ fluid of density $\rho$ permeated with neutrals of density $\rho_{d}(\ll \rho)$, and rotating about the $z$-axis with angular velocity $\Omega$, in the presence of Hall currents and oblique magnetic field. We assume that the two components of the partially ionized plasma (the ionized fluid and the neutral gas) behave as a continuum and that their steady state velocities are equal. We assume also that the magnetic field interacts only with the ionized components of the plasma and that the frictional force of the neutral gas on the ionized fluid is of the same order as the pressure gradient of the ionized fluid. The force, due to pressure gradient of the neutral gas, is much less than the frictional effects between the neutral gas and the ionized plasma in the pressure of an oblique magnetic field.
The investigation of instability problem is quite complex; consequently, we have carried out here an investigation of the instability problem using the above-stated simple model of the partially ionized plasma. The linearized perturbation equations of partially ionized plasma under the above stated assumptions are

$$
\begin{aligned}
& \rho \frac{\partial \mathbf{u}}{\partial t}=-\nabla \delta p+(\nabla \times \mathbf{h}) \times \mathbf{H}+\rho \nabla \delta P \\
&+\frac{1}{3}\left(\mu-\mu^{\prime} \frac{\partial}{\partial t}\right) \nabla(\nabla . \mathbf{u}) \\
&+\rho_{d} v_{c}\left(\mathbf{U}_{d}-\mathbf{u}\right) \\
&+\left(\mu-\mu^{\prime} \frac{\partial}{\partial t}\right) \nabla^{2} \mathbf{u}-2 \rho(\mathbf{\Omega} \times \mathbf{u}), \\
& \frac{\partial \mathbf{U}_{d}}{\partial t}=-v_{c}\left(\mathbf{U}_{d}-\mathbf{u}\right)+\nabla \delta P, \\
& \frac{\partial \mathbf{h}}{\partial t}= \nabla \times(\mathbf{u} \times \mathbf{H})+\eta \nabla^{2} \mathbf{h}-\frac{1}{N e} \nabla \\
& \times[(\nabla \times \mathbf{h}) \times \mathbf{H}], \\
& \nabla^{2} \delta P=-G \delta \rho, \\
& \frac{\partial \delta p}{\partial t}=-(\nabla \cdot \mathbf{u}) \rho, \\
& \delta p=S^{2} \delta \rho .
\end{aligned}
$$

In (1), $\rho_{d} v_{c}\left(\mathbf{U}_{d}-\mathbf{u}\right)$ represents the frictional effects of the neutral gas on the motion of the ionized fluid while $-v_{c}\left(\mathbf{U}_{d}-\mathbf{u}\right)$ in (2) represents the frictional effects of an ionized fluid on the neutral gas. In the above equations, $\mathbf{u}(u$, $v, w), \delta p, \delta \rho, \mathbf{h}\left(h_{x}, h_{y}, h_{z}\right)$, and $\delta P$ are the perturbations, respectively, in the velocity, pressure, density, magnetic field, and gravitational potential of the ionized plasma. The corresponding quantities for the neutral gas are denoted by $\mathbf{U}_{d}$ and $\rho_{d}$. The collision frequency between the two components is represented by $v_{c}$. In these equations, $e$ is the electron charge, $N$ is the number density, $\eta$ is the electrical resistivity, $\mu$ is the coefficient of viscosity, $\mu^{\prime}$ is the coefficient of viscoelasticity, and $S$ is the velocity of sound in the gas. We assume the oblique magnetic field to be uniform along the $x$ and $z$-directions $\mathbf{H}\left(H_{x}, 0, H_{z}\right)$. We seek the solution of equations (1)-(6) by assuming that all the perturbed quantities depend on $x, z$, and $t$ as

$$
\exp [i(k \sin \theta x+k \cos \theta z+n t)]
$$

where $k=(k \sin \theta, 0, k \cos \theta)$ is the wavenumber of perturbation making an angle $\theta$ with the $z$-axis and $n$ is the frequency of perturbation. On using (7) into (1)-(6) and 
eliminating $\mathbf{U}_{d}$, we obtain six equations which can be written as in what follows:

$$
\begin{gathered}
\left\{i n+\left[D+\frac{k^{2}}{3}\left(v-i n v^{\prime}\right)\right] \sin ^{2} \theta+\frac{i n \beta v_{c}}{i n+v_{c}}+k^{2}\left(v-i n v^{\prime}\right)\right\} u \\
-2 \Omega v+\left[D+\frac{k^{2}}{3}\left(\nu-i n v^{\prime}\right)\right] \cos \theta \sin \theta w \\
-\frac{i k \cos \theta}{\rho} H_{z} h_{x}+\frac{i k \sin \theta}{\rho} H_{z} h_{z}=0
\end{gathered}
$$

$$
\begin{aligned}
2 \Omega u+ & {\left[i n+k^{2}\left(\nu-i n \nu^{\prime}\right)+\frac{i n \beta v_{c}}{i n+v_{c}}\right] v } \\
& -\frac{i k}{\rho}\left(H_{x} \sin \theta+H_{z} \cos \theta\right) h_{y}=0,
\end{aligned}
$$

$$
\begin{array}{r}
{\left[D+\frac{k^{2}}{3}\left(\nu-i n \nu^{\prime}\right)\right] \cos \theta \sin \theta u} \\
+\left\{i n+\left[D+\frac{k^{2}}{3}\left(\nu-i n \nu^{\prime}\right)\right] \cos ^{2} \theta\right. \\
\left.+\frac{i n \beta v_{c}}{i n+v_{c}}+k^{2}\left(\nu-i n \nu^{\prime}\right)\right\} w \\
+\frac{i k}{\rho} H_{x} \cos \theta h_{x}-\frac{i k}{\rho} H_{x} \sin \theta h_{z}=0
\end{array}
$$

$-i k \cos \theta H_{z} u+i k \cos \theta H_{x} w+\left(i n+\eta k^{2}\right) h_{x}$

$$
\begin{aligned}
& \quad+\frac{k^{2}}{N e} \cos \theta\left(H_{z} \cos \theta+H_{x} \sin \theta\right) h_{y}=0 \\
& -i k\left(H_{x} \sin \theta+H_{z} \cos \theta\right) v \\
& -\frac{k^{2}}{N e} \cos \theta\left(H_{x} \sin \theta+H_{z} \cos \theta\right) h_{x} \\
& +\left(i n+\eta k^{2}\right) h_{y}+\frac{k^{2}}{N e} \sin \theta \\
& \quad \times\left(H_{x} \sin \theta+H_{z} \cos \theta\right) h_{z}=0
\end{aligned}
$$

$i k H_{z} \sin \theta u-i k \sin \theta H_{x} w$

$$
\begin{aligned}
& -\frac{k^{2}}{N e} \sin \theta\left(H_{x} \sin \theta+H_{z} \cos \theta\right) h_{y} \\
& +\left(i n+\eta k^{2}\right) h_{z}=0 .
\end{aligned}
$$

\section{Dispersion Relation}

Equation (8) can be written in the matrix form

$$
[A][B]=0,
$$

where $[B]$ is a single column matrix in which the elements are $\left(u, v, w, h_{x}, h_{y}, h_{z}\right)$ and $[A]$ is a sixth order matrix. The elements are

$$
\begin{aligned}
& A_{11}=i n+\left(D+\frac{k^{2}}{3}\left(\nu-i n \nu^{\prime}\right)\right) \sin ^{2} \theta \\
& +\frac{i n \beta v_{c}}{i n+v_{c}}+k^{2}\left(v-i n v^{\prime}\right), \\
& A_{12}=-2 \Omega, \quad A_{13}=\left(D+\frac{k^{2}}{3}\left(\nu-i n \nu^{\prime}\right)\right) \cos \theta \sin \theta \text {, } \\
& A_{14}=-\frac{i k \cos \theta}{\rho} H_{z}, \quad A_{15}=0, \quad A_{16}=\frac{i k \sin \theta}{\rho} H_{z}, \\
& A_{21}=2 \Omega, \quad A_{22}=i n+k^{2}\left(\nu-i n v^{\prime}\right)+\frac{i n \beta v_{c}}{i n+v_{c}}, \\
& A_{23}=A_{24}=A_{26}=0, \quad A_{25}=-\frac{i k}{\rho} L N e, \\
& A_{31}=\left[D+\frac{k^{2}}{3}\left(\nu-i n \nu^{\prime}\right)\right] \cos \theta \sin \theta \text {, } \\
& A_{32}=A_{35}=0 \\
& A_{33}=i n+\left[D+\frac{k^{2}}{3}\left(\nu-i n \nu^{\prime}\right)\right] \cos ^{2} \theta \\
& +\frac{i n \beta v_{c}}{i n+v_{c}}+k^{2}\left(v-i n v^{\prime}\right) \\
& A_{34}=\frac{i k \cos \theta}{\rho} H_{x}, \quad A_{36}=-\frac{i k \sin \theta}{\rho} H_{x}, \\
& A_{41}=-i k \cos \theta H_{z}, \quad A_{42}=A_{46}=0, \\
& A_{43}=i k \cos \theta H_{x}, \quad A_{44}=A_{66}=\left(i n+\eta k^{2}\right) \text {, } \\
& A_{45}=-A_{54}=k^{2} L \cos \theta, \quad A_{51}=A_{53}=0, \\
& A_{52}=-i k L N e, \quad A_{55}=\left(i n+\eta k^{2}\right), \\
& A_{56}=-A_{65}=k^{2} L \sin \theta, \quad A_{61}=i k \sin \theta H_{z} \text {, } \\
& A_{62}=A_{64}=0, \quad A_{63}=-i k \sin \theta H_{x},
\end{aligned}
$$

where we have written

$$
D=\frac{s^{2} k^{2}}{i n}-\frac{G \rho(1+\beta)}{i n}+\frac{G \rho \beta}{i n+v_{c}}, \quad \beta=\frac{\rho_{d}}{\rho} \text {, }
$$


The vanishing of $|A|$ gives the following relation as the product

$$
\begin{aligned}
& \left(i n+\eta k^{2}\right)\left\{\left[\left(i n+\eta k^{2}\right)^{2}+k^{4} L^{2}\right]\right. \\
& \times\left[i n+\frac{i n \beta v_{c}}{i n+v_{c}}+k^{2}\left(\nu-i n \nu^{\prime}\right)\right]^{3} \\
& +\left[i n+\frac{i n \beta v_{c}}{i n+v_{c}}+k^{2}\left(v-i n v^{\prime}\right)\right]^{2} \\
& \times\left[\left(D+\frac{k^{2}}{3}\left(\nu-i n v^{\prime}\right)\right)\left[\left(i n+\eta k^{2}\right)^{2}+k^{4} L^{2}\right]\right. \\
& +\frac{k^{2}\left(i n+\eta k^{2}\right)}{\rho} \\
& \left.\times\left[\left(H_{x} \cos \theta-H_{z} \sin \theta\right)^{2}-2 L^{2}(N e)^{2}\right]\right] \\
& +\left[i n+\frac{i n \beta v_{c}}{i n+v_{c}}+k^{2}\left(\nu-i n v^{\prime}\right)\right] \\
& \times\left[\frac{2 k^{2}\left(i n+\eta k^{2}\right)}{\rho}\left(D+\frac{k^{2}}{3}\left(\nu-i n \nu^{\prime}\right)\right)\right. \\
& +4 \Omega^{2}\left[\left(i n+\eta k^{2}\right)^{2}+k^{4} L^{2}\right] \\
& +\frac{4 k^{4} L \Omega H_{z}}{\rho}\left[H_{x} \sin \theta+H_{z} \cos \theta\right] \\
& \left.+\frac{k^{4}\left(H_{x}^{2}+H_{z}^{2}\right)}{\rho^{2}}\left(H_{x} \sin \theta+H_{z} \cos \theta\right)^{2}\right] \\
& +\left[D+\frac{k^{2}}{3}\left(\nu-i n v^{\prime}\right)\right] \\
& \times\left[4 \Omega^{2}\left(i n+\eta k^{2}\right)^{2} \cos ^{2} \theta+4 k^{4} L^{2} \Omega^{2} \cos ^{2} \theta\right] \\
& +4 k L \rho^{-1} \Omega \cos \theta L^{2}(N e)^{2} \\
& \times\left\{k ^ { 4 } \rho ^ { - 2 } \left[\left(H_{x}^{2} \sin ^{2} \theta-H_{z}^{2} \cos ^{2} \theta\right)^{2}\right.\right. \\
& \left.\left.+4 H_{x} H_{z} \cos \theta \sin \theta L^{2}(N e)^{2}\right]\right\} \\
& \left.+4 \Omega^{2} H_{x}^{2} \frac{k^{2}}{\rho}\left(i n+\eta k^{2}\right)\right\}=0 .
\end{aligned}
$$

The first factor of equation (12) gives

$$
n=i \eta k^{2}
$$

which corresponds to viscous type of damped mode modified by finite conductivity. By writing $n=i W$ and using the value of $D$ in the second factor of equation (12), we obtain the resulting dispersion relation which is an equation of the tenth degree in $W$ of the form

$$
\begin{aligned}
d_{0} W^{10}- & d_{1} W^{9}+d_{2} W^{8}-d_{3} W^{7}+d_{4} W^{6}-d_{5} W^{5} \\
& +d_{6} W^{4}-d_{7} W^{3}+d_{8} W^{2}-d_{9} W+d_{10}=0,
\end{aligned}
$$

where the coefficients $d_{0}-d_{10}$ in (14) can obviously be obtained from (12). We state here explicitly only the coefficients $d_{0}$ and $d_{10}$, since we discuss the nature of the roots of $W$ with their help, that is,

$$
\begin{aligned}
d_{0}= & \frac{1}{3}\left(k^{2} v^{\prime}-1\right)^{2}\left(3-4 k^{2} \nu^{\prime}\right), \\
d_{10}= & \frac{k^{6} \eta v_{c}^{3}}{\rho^{2}}\left[k^{2} S^{2}-G \rho(1+\beta)\right] \\
& \times\left\{\rho^{2}\left(L^{2}+\eta^{2}\right)\left(k^{4} v^{2}+4 \Omega^{2} \cos ^{2} \theta\right)\right. \\
& +2 \rho\left(H_{x} \sin \theta+H_{z} \cos \theta\right) \\
& \times\left(k^{2} \eta \nu+2 L \Omega \cos \theta\right) \\
& \left.+\left(H_{x}^{2} \sin ^{2} \theta-H_{z}^{2} \cos ^{2} \theta\right)^{2}\right\}
\end{aligned}
$$

and the coefficients $d_{2}-d_{9}$ are not given here because they are very lengthy.

\section{Stability Analysis and Discussion}

Equation (15) shows that when $3-4 k^{2} v^{\prime} \gtrless 0$ and $k^{2} S^{2}-$ $G \rho(1+\beta)<0$, then the product of the roots is negative. Therefore, equation (14) has always one negative real root. The considered plasma is, therefore, unstable when $k<$ $\sqrt{G \rho(1+\beta)} / S$, which is precisely the Jeans' criterion for a partially ionized plasma, irrespective of the kinematic viscoelasticity value. However, (15) shows also that when $3-4 k^{2} v^{\prime}>0$ and $k^{2} S^{2}-G \rho(1+\beta)>0$, that is, for the wavenumber range $(1 / 2) \sqrt{3 / \nu^{\prime}}>k>\sqrt{G \rho(1+\beta)} / S$, then the product of roots is positive. Equation (14) has, therefore, either all positive real roots or pairs of complex conjugate roots. The real roots correspond to stable modes. The complex roots also correspond to stable modes, since $\operatorname{Re}(W)$ is always positive as the equation satisfied by $\operatorname{Re}(W)$ turns out to be one which has its coefficients alternatively positive and negative. Hence, Jeans' criterion for gravitational stability $k>\sqrt{G \rho(1+\beta)} / S$ remains unchanged in the presence of the effects of magnetic resistivity, Hall currents, and ion viscosity when the plasma is permeated by an oblique magnetic field. In addition to this usual Jeans' criterion for stability there exists a new stability condition which occurs for wavenumber values $k<(1 / 2) \sqrt{3 / v^{\prime}}$ depends on the kinematic viscoelasticity appears due to the viscoelastic Walters B' plasma model.

In order to study the influences of various physical parameters on the growth rate of an unstable mode, we have performed numerical calculations of the dispersion relation (14), using Mathematica 6, to locate the roots of 


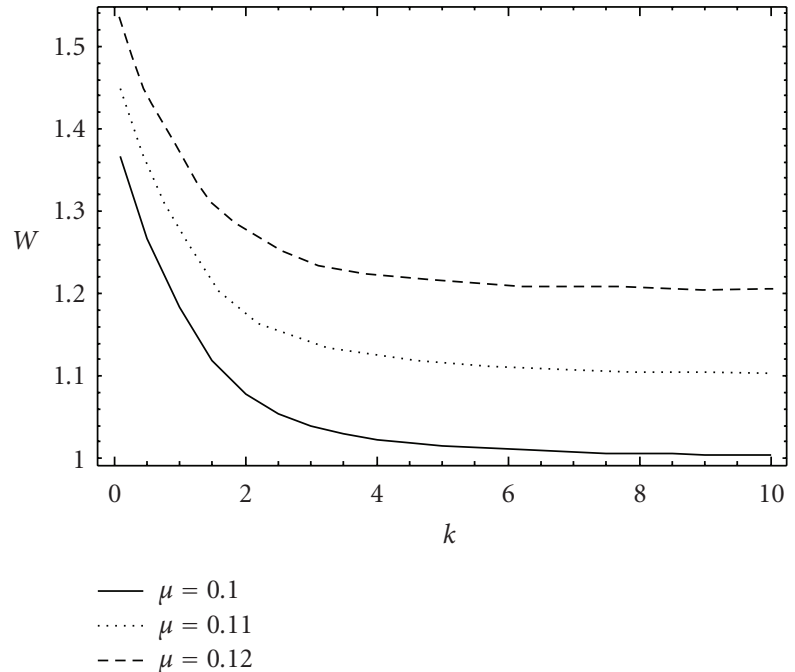

FIGURE 1: Variation of $W$, given by (14), with the wavenumber for different values of viscosity $\mu$ when $\mu^{\prime}=0.1 \mathrm{gm} \mathrm{cm}^{-1} \mathrm{sec}^{-2}, \eta=$ $1 \mathrm{mH} \mathrm{cm}{ }^{-1}, v_{c}=0.1 \mathrm{sec}^{-1}, \Omega=0.05 \mathrm{rad} \mathrm{sec}^{-1}, H_{z}=0.1 \mathrm{Amp} \mathrm{cm}^{-1}$, $\rho_{d}=0.001 \mathrm{gm} \mathrm{cm}^{-3}, L=0.1 \mathrm{~cm}^{2} \mathrm{sec}^{-1}$, and $\theta=0^{\circ}$.

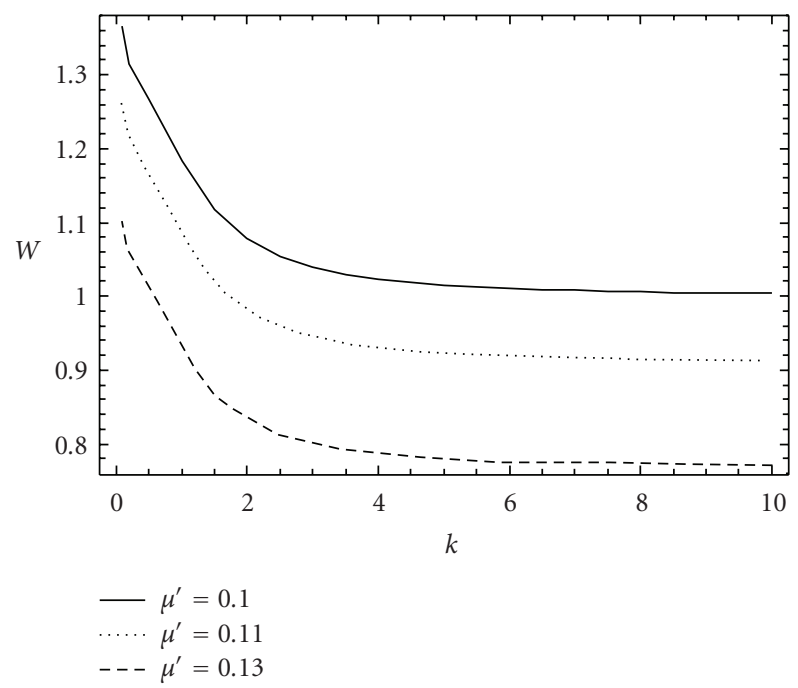

FIGURE 2: Variation of $W$, given by (14), with the wavenumber for different values of viscoelasticity $\mu^{\prime}$ when $\eta=1 \mathrm{mH} \mathrm{cm}^{-1}$, $\mu=0.1 \mathrm{gm} \mathrm{cm}^{-1} \mathrm{sec}^{-1}, v_{c}=0.1 \mathrm{sec}^{-1}, \Omega=0.05 \mathrm{rad} \mathrm{sec}^{-1}, H_{z}=$ $0.1 \mathrm{Amp} \mathrm{cm}{ }^{-1}, \rho_{d}=0.001 \mathrm{gm} \mathrm{cm}^{-3}, L=0.1 \mathrm{~cm}^{2} \mathrm{sec}^{-1}$, and $\theta=0^{\circ}$.

the growth rate $W$ against the wavenumber $k$ for various values of the parameters included in the analysis. For these calculations, we take the numerical values for the following physical quantities which correspond to the conditions in galaxies Bhatia and Hazarika [24]): $\rho=1.7 \times 10^{-21} \mathrm{~kg} \mathrm{~m}^{-3}$, $G=6.658 \times 10^{-11}(\mathrm{~kg})^{-1} \mathrm{~m}^{3} \mathrm{~s}^{-2}$, and $S^{2}=2.5 \times 10^{8} \mathrm{~m}^{2} \mathrm{~s}^{-2}$. These calculations are presented in Figures 1-5 and Tables 14 , to show the variation of the growth rate with wavenumber of the considered system for different values of viscosity $\mu$, viscoelasticity $\mu^{\prime}$, electrical resistivity $\eta$, density of neutral particles $\rho_{d}$, the magnetic field component $H_{z}$ in the $z$-direction, inclination angle $\theta$ (between the wavenumber

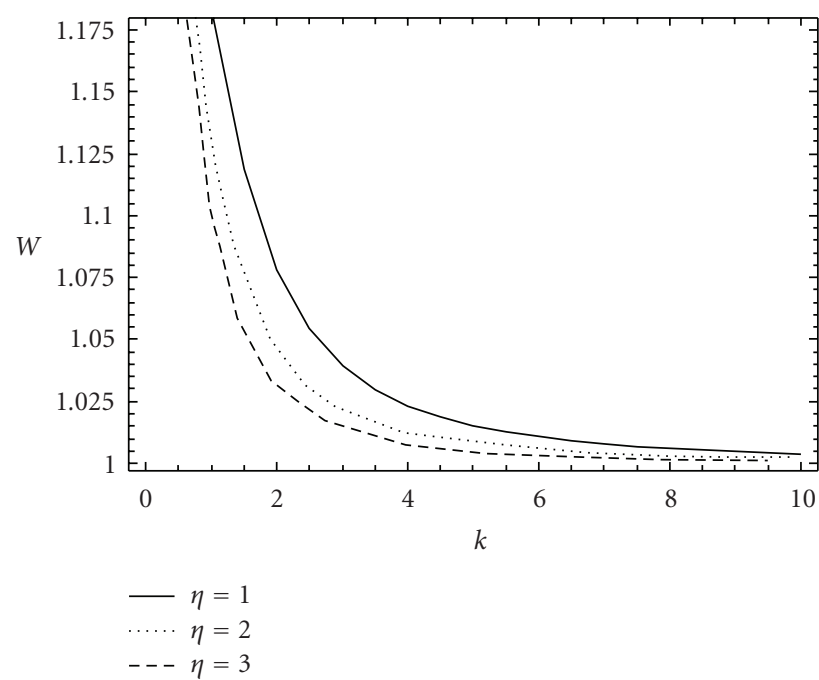

Figure 3: Variation of $W$, given by (14), with the wavenumber for different values of electrical resistivity $\eta$ when $\mu^{\prime}=$ $0.1 \mathrm{gm} \mathrm{cm}^{-1} \mathrm{sec}^{-2}, \mu=0.1 \mathrm{gm} \mathrm{cm}^{-1} \mathrm{sec}^{-1}, v_{c}=0.1 \mathrm{sec}^{-1}, \Omega=$ $0.05 \mathrm{rad} \mathrm{sec}^{-1}, H_{z}=0.1 \mathrm{Amp} \mathrm{cm}^{-1}, \rho_{d}=0.001 \mathrm{gm} \mathrm{cm}^{-3}, L=$ $0.1 \mathrm{~cm}^{2} \mathrm{sec}^{-1}$, and $\theta=0^{\circ}$.

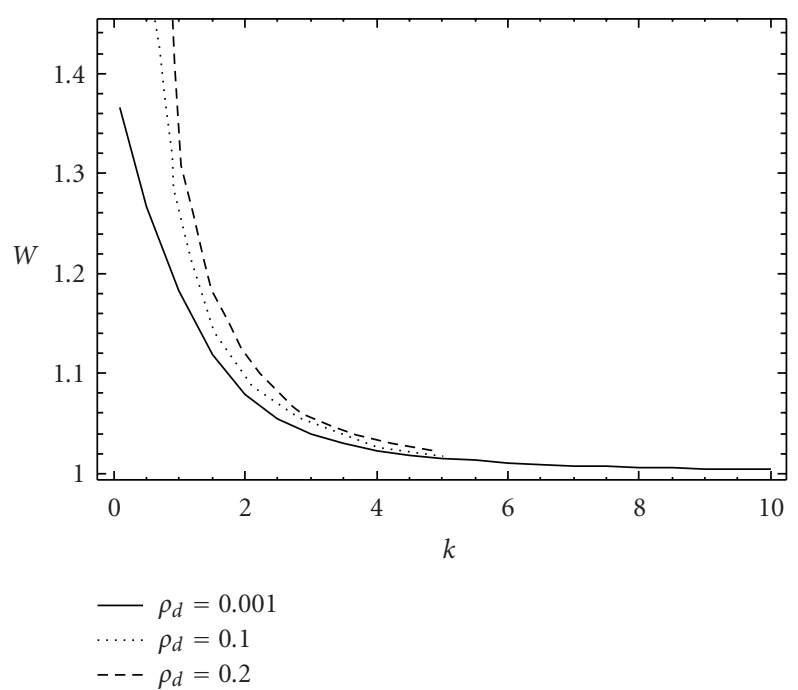

FIgURE 4: Variation of $W$, given by (14), with the wavenumber for different values of $\rho_{d}$ when $\mu^{\prime}=0.1 \mathrm{gm} \mathrm{cm}^{-1} \mathrm{sec}^{-2}, \eta=1 \mathrm{mH} \mathrm{cm}^{-1}$, $v_{c}=0.1 \mathrm{sec}^{-1}, \Omega=0.05 \mathrm{rad} \mathrm{sec}^{-1}, H_{z}=0.1 \mathrm{Amp} \mathrm{cm}^{-1}, \mu=$ $0.1 \mathrm{gm} \mathrm{cm}^{-1} \mathrm{sec}^{-1}, L=0.1 \mathrm{~cm}^{2} \sec ^{-1}$ and $\theta=0^{\circ}$.

vector and the $z$-axis), Hall currents $L$, angular frequency of rotation $\Omega$, and collisions with neutrals $v_{c}$, respectively.

Figure 1 shows the variation of the growth rate $W$ with the wavenumber $k$ for various values of viscosity $\mu$ and indicates that the growth rate $W$ increases by increasing the viscosity $\mu$ for the same $\theta$ and $k$, showing thereby the stabilizing effect of the viscosity $\mu$. Figure 2 shows the variation of the growth rate $W$ with wavenumber $k$ for various values of viscoelasticity $\mu^{\prime}$ and indicates that the growth rate $W$ decreases by increasing the viscoelasticity $\mu^{\prime}$ for the same $\theta$ and $k$, showing thereby the destabilizing influence of the viscoelasticity $\mu^{\prime}$. Figure 3 shows the variation of the growth 
TABLE 1: Variation of $W$, given by (14), with the wavenumber for different values of $\theta$. At $\mu^{\prime}=0.1 \mathrm{gm} \mathrm{cm}^{-1} \mathrm{sec}^{-2}, \mu=0.1 \mathrm{gm} \mathrm{cm}^{-1} \mathrm{sec}^{-1}$, $\eta=1 \mathrm{mH} \mathrm{cm}{ }^{-1}, v_{c}=0.1 \mathrm{sec}^{-1}, \Omega=0.05 \mathrm{rad} \mathrm{sec}^{-1}, \rho_{d}=0.001 \mathrm{gm} \mathrm{cm}^{-3}, L=0.1 \mathrm{~cm}^{2} \mathrm{sec}^{-1}$, and $H_{z}=0.1 \mathrm{Amp} \mathrm{cm}^{-1}$.

\begin{tabular}{lccccc}
\hline$k$ & $\theta=0^{\circ}$ & $\theta=30^{\circ}$ & $\theta=45^{\circ}$ & $\theta=60^{\circ}$ & $\theta=90^{\circ}$ \\
\hline 0.1 & 1.36664 & 1.41271 & 1.43026 & 1.43829 & 1.37685 \\
0.2 & 1.31426 & 1.35344 & 1.36885 & 1.3568 & 1.36133 \\
0.3 & 1.2968 & 1.3341 & 1.3488 & 1.34996 & 1.34164 \\
0.4 & 1.28205 & 1.31794 & 1.33196 & 1.32222 & 1.30311 \\
0.5 & 1.26666 & 1.30106 & 1.31422 & 1.28289 & 1.30795 \\
0.6 & 1.25032 & 1.28308 & 1.29511 & 1.26193 & 1.2703 \\
0.7 & 1.23339 & 1.26431 & 1.27489 & 1.23951 & 1.25073 \\
0.8 & 1.21631 & 1.24528 & 1.25393 & 1.22132 \\
0.9 & 1.19955 & 1.22648 & 1.23151 & 1.23135 \\
1 & 1.18344 & 1.20832 & 1.21332 & \\
\hline
\end{tabular}

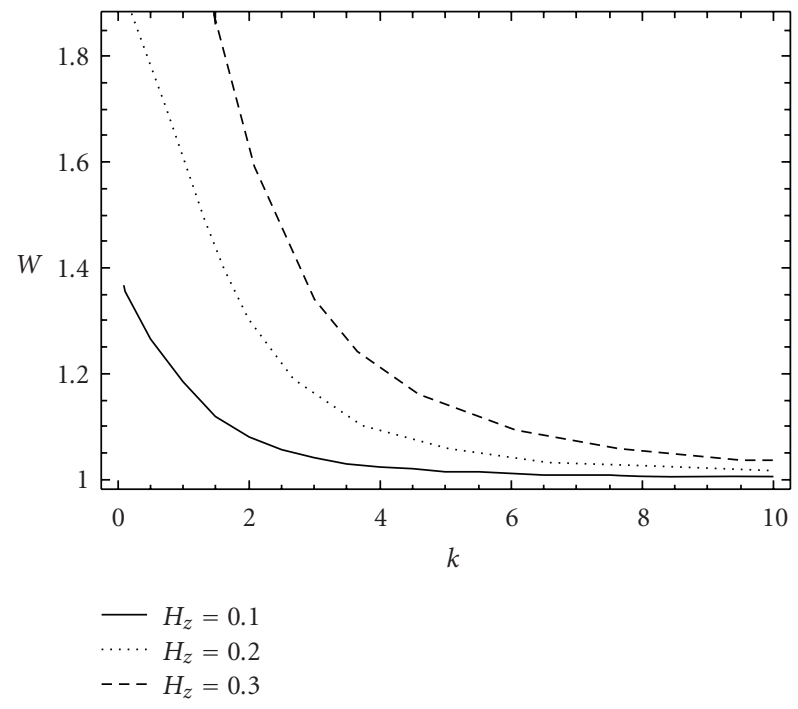

FIGURE 5: Variation of $W$, given by (14), with the wavenumber for different values of magnetic field in $z$ direction $H_{z}$ when $\mu^{\prime}=0.1 \mathrm{gm} \mathrm{cm}^{-1} \mathrm{sec}^{-2}, \eta=1 \mathrm{mH} \mathrm{cm}^{-1}, v_{c}=0.1 \mathrm{sec}^{-1}, \Omega=$ $0.05 \mathrm{rad} \mathrm{sec}^{-1}, \mu=0.1 \mathrm{gm} \mathrm{cm}^{-1} \mathrm{sec}^{-1}, \rho_{d}=0.001 \mathrm{gm} \mathrm{cm}^{-3}, L=$ $0.1 \mathrm{~cm}^{2} \sec ^{-1}$ and $\theta=0^{\circ}$.

rate $W$ with the wavenumber $k$ for various values of the electrical resistivity $\eta$ and indicates, for the same $\theta$ and $k$, that the growth rate $W$ decreases by increasing the electrical resistivity $\eta$ only for small wavenumber values, showing thereby its destabilizing effect for small wavenumbers, while electrical resistivity $\eta$ is found to has a slightly destabilizing influence for higher wavenumber values. Figure 4 illustrates the behavior of the growth rate $W$ with the wavenumber $k$ for various values of the density of neutral particles $\rho_{d}$. It is clear from Figure 4 that the growth rate $W$ increases by increasing the density of neutral particles $\rho_{d}$ only for small wavenumber values less than 5 , showing thereby its stabilizing effect for the wavenumbers range $0<k<5$, while it shows also that the density of neutral particles $\rho_{d}$ has no effect on the stability of the considered system for wavenumber values $k>5$. Also, Figure 5 illustrates the behavior of the growth rate $W$ with the wavenumber $k$ for
TABle 2: Variation of $W$, given by (14), with the wavenumber for different values of $L$. At $\mu^{\prime}=0.1 \mathrm{gm} \mathrm{cm}^{-1} \mathrm{sec}^{-2}, \mu=$ $0.1 \mathrm{gm} \mathrm{cm}^{-1} \mathrm{sec}^{-1}, \quad \eta=1 \mathrm{mH} \mathrm{cm}{ }^{-1}, \quad v_{c}=0.1 \mathrm{sec}^{-1}, \Omega=$ $0.05 \mathrm{rad} \mathrm{sec}^{-1}, \rho_{d}=0.001 \mathrm{gm} \mathrm{cm}^{-3}, \theta=0^{\circ}$, and $H_{z}=$ $0.1 \mathrm{Amp} \mathrm{cm}^{-1}$.

\begin{tabular}{lccc}
\hline$k$ & $L=0.1$ & $L=0.5$ & $L=1$ \\
\hline 0.1 & 1.36664 & 1.36663 & 1.36662 \\
0.2 & 1.31426 & 1.31419 & 1.31398 \\
0.3 & 1.2968 & 1.29648 & 1.29547 \\
0.4 & 1.28205 & 1.28113 & 1.27828 \\
0.5 & 1.26666 & 1.2647 & 1.25866 \\
0.6 & 1.25032 & 1.2469 & 1.23638 \\
0.7 & 1.23339 & 1.22814 & 1.21228 \\
0.8 & 1.21631 & 1.20908 & 1.18765 \\
0.9 & 1.19955 & 1.19034 & 1.16375 \\
1 & 1.18344 & 1.17244 & 1.14156 \\
\hline
\end{tabular}

various values of the magnetic field component $H_{z}$, and it shows that the growth rate $W$ increases by increasing the magnetic field component $H_{z}$ in the $z$-direction only for small wavenumber values less than 10 , showing thereby its stabilizing effect for such wavenumbers, while it shows also that $H_{z}$ has a slightly stabilizing effect afterwards for wavenumber values $k>10$. Table 1 shows the changes of the growth rate $W$ with the wavenumber $k$ for various values of the inclination angle $\theta$. It is clear from this table that the growth rate $W$ increases by increasing the inclination angle $\theta$ (between the wavenumber vector and the $z$-axis), showing thereby the stabilizing effect of the inclination angle $\theta$. It is clear also that the growth rate $W$ for longitudinal disturbances (i.e., when $\theta=\pi / 2$ ) is larger than its value for transverse disturbances (i.e., when $\theta=0$ ), which means that the system is stable in the first case faster than in the later case. Also, it can be seen from Tables 2 and 3 that the growth rates $W$ slightly decrease by increasing the Hall current parameter $L$ as well as the angular frequency of rotation $\Omega$, showing thereby the slightly destabilizing effects of the Hall current $L$ and the rotation $\Omega$. Finally, Table 4 indicates that the growth rate $W$ increases by increasing the collisions with neutrals parameter $v_{c}$, which means that the collisions with 
TABle 3: Variation of $W$, given by (14), with the wavenumber for different values of $\Omega$. At $\mu^{\prime}=0.1 \mathrm{gm} \mathrm{cm}^{-1} \mathrm{sec}^{-2}, \mu=$ $0.1 \mathrm{gm} \mathrm{cm}^{-1} \mathrm{sec}^{-1}, \quad \eta=1 \mathrm{mH} \mathrm{cm}{ }^{-1}, \quad v_{c}=0.1 \mathrm{sec}^{-1}, L=$ $0.1 \mathrm{~cm}^{2} \mathrm{sec}^{-1}, \rho_{d}=0.001 \mathrm{gm} \mathrm{cm}^{-3}, \theta=0^{\circ}$, and $H_{z}=$ $0.1 \mathrm{Amp} \mathrm{cm}^{-1}$.

\begin{tabular}{lccc}
\hline$k$ & $\Omega=0.05$ & $\Omega=10^{18}$ & $\Omega=10^{19}$ \\
\hline 0.1 & 1.36664 & 1.31438 & 1.29901 \\
0.2 & 1.31426 & 1.30745 & 1.25293 \\
0.3 & 1.2968 & 1.29528 & 1.25156 \\
0.4 & 1.28205 & 1.28146 & 1.25063 \\
0.5 & 1.26666 & 1.26633 & 1.24872 \\
0.6 & 1.25032 & 1.2501 & 1.23963 \\
0.7 & 1.23339 & 1.23321 & 1.22644 \\
0.8 & 1.21631 & 1.21616 & 1.21143 \\
0.9 & 1.19955 & 1.19942 & 1.19589 \\
1 & 1.18344 & 1.18332 & 1.18056 \\
\hline
\end{tabular}

TABLE 4: Variation of $W$, given by (14), with the wavenumber for different values of $v_{c}$. At $\mu^{\prime}=0.1 \mathrm{gm} \mathrm{cm}^{-1} \mathrm{sec}^{-2}, \mu=$ $0.1 \mathrm{gm} \mathrm{cm}^{-1} \mathrm{sec}^{-1}, \eta=1 \mathrm{mH} \mathrm{cm}{ }^{-1}, \Omega=0.05 \mathrm{rad} \mathrm{sec}^{-1}, L=$ $0.1 \mathrm{~cm}^{2} \mathrm{sec}^{-1}, \rho_{d}=0.001 \mathrm{gm} \mathrm{cm}^{-3}, \theta=0^{\circ}$, and $H_{z}=$ $0.1 \mathrm{Amp} \mathrm{cm}^{-1}$.

\begin{tabular}{lccc}
\hline$k$ & $v_{c}=0.1$ & $v_{c}=0.1$ & $v_{c}=0.2$ \\
\hline 0.1 & 1.36664 & 1.423 & 1.47494 \\
0.2 & 1.31426 & 1.32782 & 1.33983 \\
0.3 & 1.2968 & 1.30284 & 1.30814 \\
0.4 & 1.28205 & 1.28547 & 1.28847 \\
0.5 & 1.26666 & 1.26887 & 1.27081 \\
0.6 & 1.25032 & 1.25188 & 1.25325 \\
0.7 & 1.23339 & 1.23455 & 1.23556 \\
0.8 & 1.21631 & 1.21722 & 1.218 \\
0.9 & 1.19955 & 1.20027 & 1.20089 \\
1 & 1.18344 & 1.18403 & 1.18454 \\
\hline
\end{tabular}

neutrals $v_{c}$ has a stabilizing effect. Finally, it should be noted that these results are in agreement with earlier observations of Chhonkar and Bhatia [25], Ali and Bhatia [23], and Bhatia and Hazarika [24] in absence of kinematic viscoelasticity, and the additional behaviors of other physical parameters arose from the presence of viscoelasticity due to the used viscoelastic Walters $B^{\prime}$ model.

\section{Conclusion}

The gravitational instability of a rotating Walters B' partially ionized plasma permeated by an oblique magnetic field has been investigated in the presence of the effects of Hall currents, electrical resistivity, and ion viscosity. The dispersion relation is obtained and numerical calculations have been performed to obtain the dependence of the growth rate of the gravitational unstable mode on the various physical effects. We found that

(1) viscosity and collision frequency of the two component partially ionized plasma have stabilizing effects,
(2) viscoelasticity and angular frequency of rotation have destabilizing effects,

(3) the electrical resistivity has a destabilizing effect only for small wavenumbers, while it has a slightly destabilizing influence for higher wavenumber values,

(4) the density of neutral particles $\rho_{d}$ only for small wavenumber values less than 5 , showing thereby its stabilizing effect for the wavenumbers range $0<$ $k<5$, while it has no effect on the stability of the considered system for wavenumber values $k>5$,

(5) the magnetic field component $H_{z}$ in the $z$-direction has a stabilizing effect only for small wavenumber values less than 10 , while it has a slightly stabilizing effect afterwards for $k>10$,

(6) the Hall current parameter has a slightly destabilizing effect,

(7) the increase in the angle $\theta$ (between the wavenumber of perturbation and he $z$-direction) has a destabilizing effect on the stability of the considered system for all physical parameters.

\section{Acknowledgment}

The authors would like to thank Professor M. A. Kamel (Ain Shams University, Egypt) for his critical reading of the manuscript and for his valuable comments and discussion.

\section{References}

[1] J. H. Jeans, "The stability of spherical nebula," Philosophical Transactions of the Royal Society, vol. 199, no. 7, pp. 1-53, 1902.

[2] S. Chandrasekhar, Hydrodynamic and Hydromagnetic Stability, Dover, New York, NY, USA, 1981.

[3] P. K. Bhatia, "Influence of finite larmor radius and finite conductivity on the gravitational instability of a plasma," Nuovo Cimento B, vol. 59, no. 2, pp. 228-235, 1969.

[4] C. P. Yu and J. V. Sanborn, "Internal gravitational instability in a stratified anisotropic plasma," Physics of Fluids, vol. 10, no. 11, pp. 2436-2441, 1967.

[5] P. K. Bhatia, "Gravitational instability of a rotating anisotropic plasma," Physics of Fluids, vol. 10, no. 8, pp. 1652-1653, 1967.

[6] E. P. Barbian and C. E. Rasmussen, "Rotating plasma and gravitational instability," Plasma Physics, vol. 11, no. 3, pp. 197-210, 1969.

[7] P. D. Ariel, "Gravitational instability of a rotating anisotropic plasma with Hall's effect," Physics of Fluids, vol. 13, no. 6, pp. 1644-1646, 1970.

[8] A. A. Mamun and P. K. Shukla, "A new magnetic Jeans instability in a nonuniform partially ionized plasma," Physica Scripta, vol. 62, no. 5, pp. 429-432, 2000.

[9] B. D. Pandey, B. Van der Holst, J. Vranjes, and S. Poedts, "Jeans instability of an inhomogenous streaming dusty plasma," Pramana-Jouranal of Physics, vol. 61, no. 1, pp. 109-120, 2003.

[10] W. Daughton, P. J. Catto, B. Coppi, and S. I. Krasheninnikov, "Interchange instabilities in a partially ionized plasma," Physics of Plasmas, vol. 5, no. 6, pp. 2217-2231, 1998.

[11] A. A. Mamun, "Effects of dust temperature and fast ions on gravitational instability in a self-gravitating magnetized dusty 
plasma," Physics of Plasmas, vol. 5, no. 10, pp. 3542-3546, 1998.

[12] A. A. Mamun and P. K. Shukla, "Drift-like dust-convective cells in a dusty magnetoplasma," Physics of Plasmas, vol. 7, no. 11, pp. 4766-4769, 2000.

[13] A. A. Mamun and P. K. Shukla, "Shear Alfvén-like waves in a weakly ionized self-gravitating nonuniform dusty magnetoplasma," Physics of Plasmas, vol. 8, no. 7, p. 3513, 2001.

[14] M. C. De Juli, D. Falceta-Gonçalves, and V. Jatenco-Pereira, "Alfvén waves propagation in homogeneous and dusty astrophysical plasmas," Advances in Space Research, vol. 35, no. 5, pp. 925-935, 2005.

[15] N. F. Cramer and F. Verheest, "The Alfven and compressive resonances in adusty self-gravitating plasma," Physics of Plasmas, vol. 12, Article ID 082902, 2005.

[16] M. Azeem and A. M. Mirza, "Self-gravitation effect on the nonlinear dynamics of an electron-positron-ion magnetoplasma," Physica Scripta, vol. 73, no. 1, pp. 120-124, 2006.

[17] M. F. El-Sayed and R. A. Mohamed, "Magnetogravitational instability of thermally conducting rotating viscoelastic fluid with Hall current in Brinkman porous medium," Journal of Porous Media, vol. 13, no. 9, pp. 779-798, 2010.

[18] R. C. Sharma, . Sunil, and S. Chand, "The instability of streaming Walters' viscoelastic fluid $\mathrm{B}^{\prime}$ in porous medium," Czechoslovak Journal of Physics, vol. 49, no. 2, pp. 189-195, 1999.

[19] P. Kumar, "Stability of two superposed viscoelastic (Walters B') fluid-particle mixtures in porous medium," Zeitschrift fur Naturforschung A, vol. 54, no. 5, pp. 343-347, 1999.

[20] P. Kumar, "Thermal convection in Walters B' viscoelastic fluid permeated with suspended particles in porous medium," Indian Journal of Pure and Applied Mathematics, vol. 30, no. 11, pp. 1117-1123, 1999.

[21] R. K. Chhajlani and M. K. Vyas, "Kelvin-Helmholtz instabolity of composite plasma in an oblique magnetic field with resistivity," Astrophysics and Space Science, vol. 173, no. 1, pp. 109-125, 1990.

[22] A. Khan and P. K. Bhatia, "Gravitational instability of a rotating fluid in an oblique magnetic field," Physica Scripta, vol. 47, no. 2, pp. 230-234, 1993.

[23] A. Ali and P. K. Bhatia, "Gravitational instability of partiallyionized plasma in an oblique magnetic field," Astrophysics and Space Science, vol. 195, no. 2, pp. 389-400, 1992.

[24] P. K. Bhatia and A. B. R. Hazarika, "Gravitational instability of partiallyionized plasma in an oblique magnetic field," Physica Scripta, vol. 51, no. 6, pp. 775-779, 1995.

[25] R. P. S. Chhonkar and P. K. Bhatia, "Larmor radius effects on the gravitational instability of a two-component plasma," Journal of Plasma Physics, vol. 18, no. 2, pp. 273-286, 1977. 

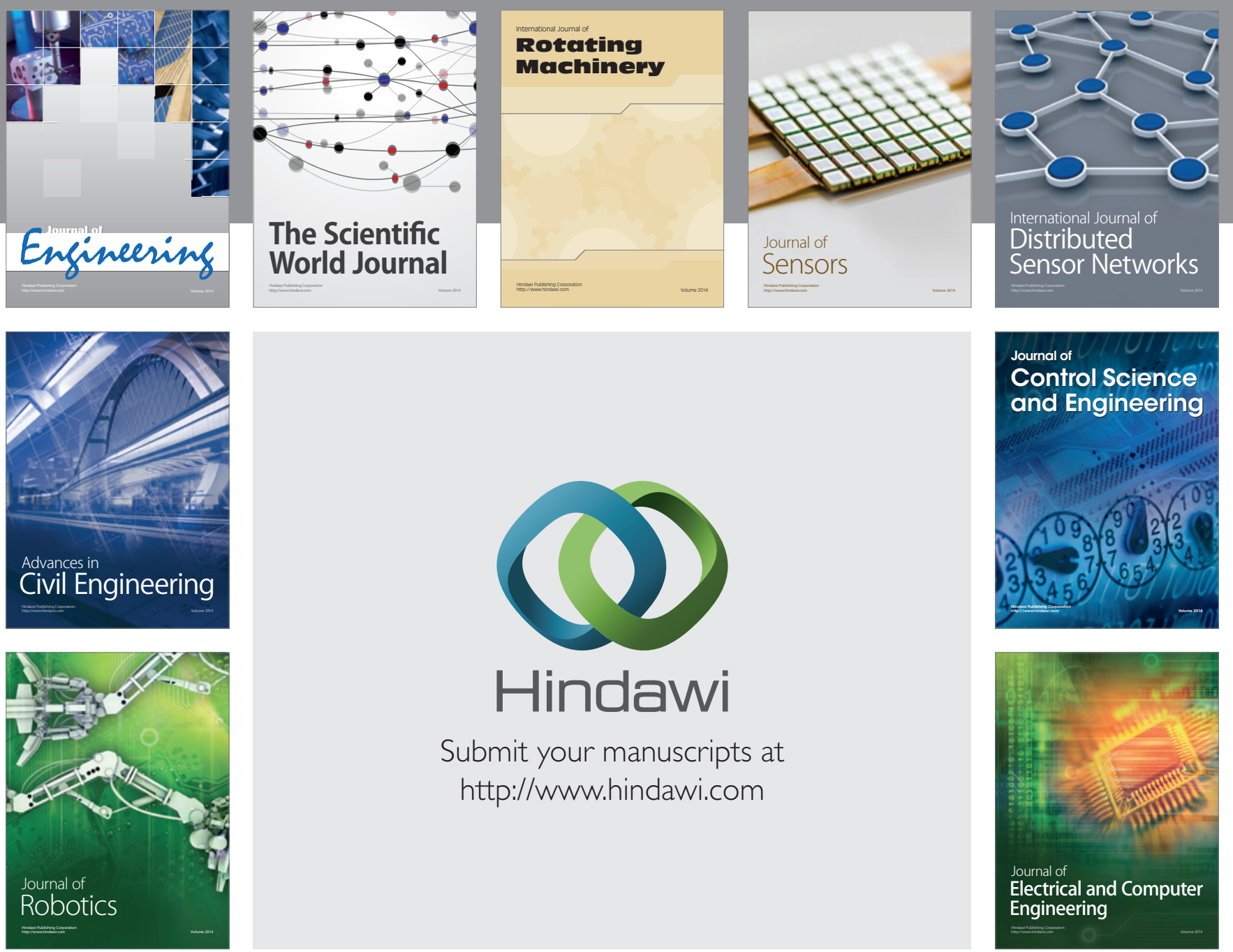

Submit your manuscripts at

http://www.hindawi.com
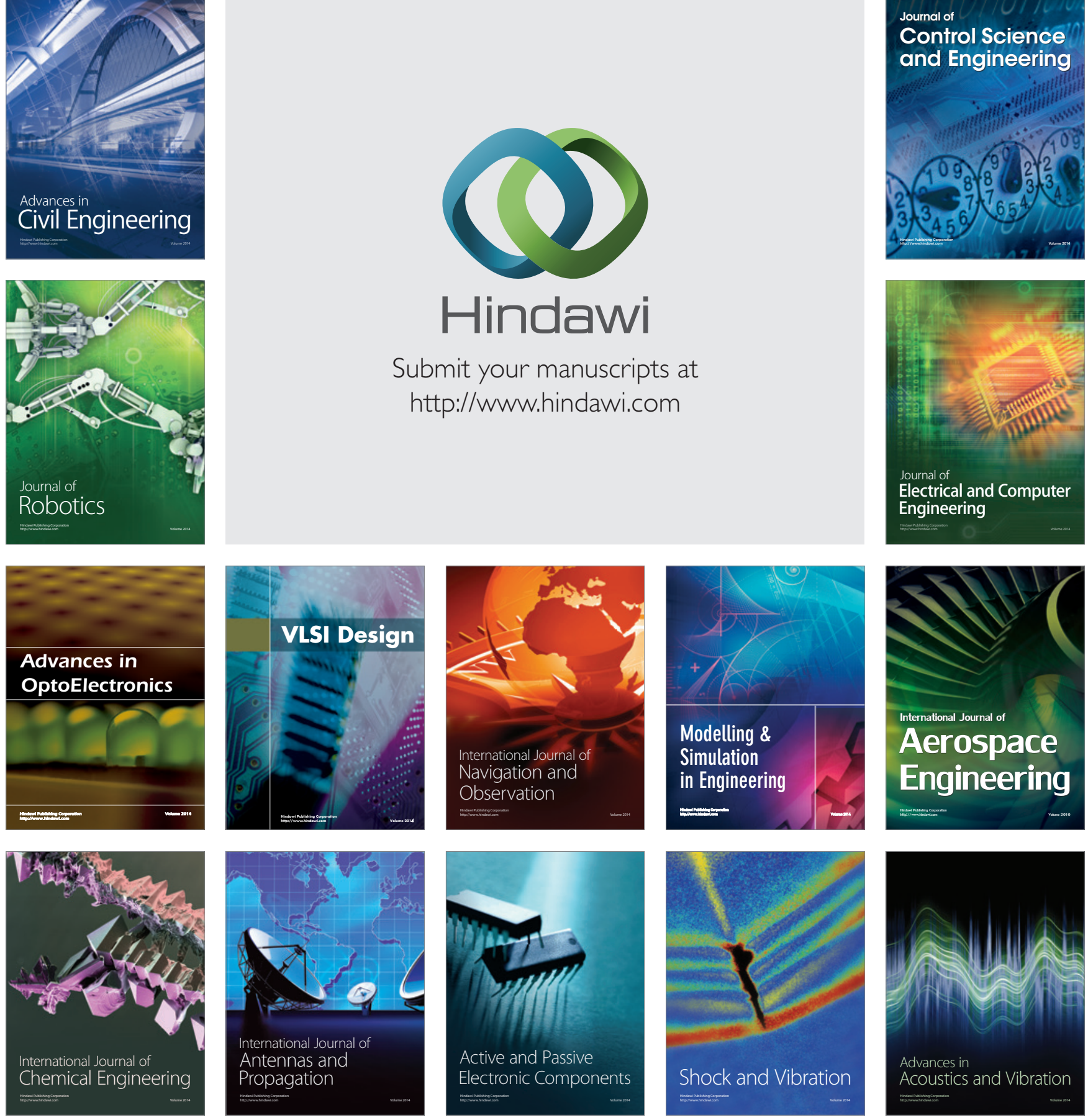